\title{
Aprimorando e validando um fotogate de baixo custo
}

\author{
Enhancing and validating a low-cost photogate
}

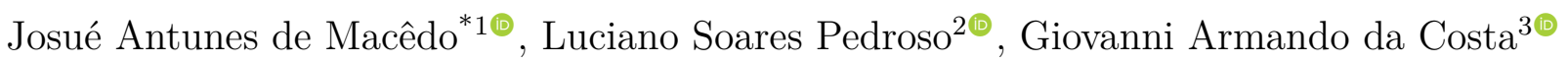 \\ ${ }^{1}$ Instituto Federal de Educação, Ciência e Tecnologia do Norte de Minas Gerais, Campus Januária, Januária, MG, Brasil \\ ${ }^{2}$ Universidade Federal dos Vales do Jequitinhonha e Mucuri, Licenciatura em Educação do Campo, Diamantina, MG, Brasil \\ ${ }^{3}$ Universidade do Estado de Minas Gerais, Unidade de Passos, Passos, MG, Brasil
}

Recebido em 06 de Setembro, 2017. Revisado em 15 de Março, 2018. Aceito em 12 de Abril, 2018.

\begin{abstract}
Este trabalho apresenta o desenvolvimento e validação de um instrumento de baixo custo que pode ser utilizado para medidas de intervalos de tempo, e em outros experimentos de física. Propõe-se a construção de um fotogate aliando a simplicidade e a economia, o que o torna mais simples e barato do que aqueles que se encontram no mercado. A construção desse instrumento baseia-se na utilização de um resistor dependente de luz (LDR) e um apontador LASER, com conexões simples e elementares o que o caracteriza como instrumento de medida de baixo custo. A sua validação ocorreu através da comparação entre os resultados do momento de inércia de uma barra homogênea e delgada e os experimentos realizados em um laboratório, comprovando sua efetividade como instrumento de coleta de variação de tempo. Propõe-se ainda uma atividade experimental, cujo objetivo é determinar o ponto de aterrissagem de uma esfera lançada horizontalmente do tampo de uma mesa, a uma velocidade qualquer. O seu uso tende a facilitar a difusão e o acesso a este tipo de instrumento de medidas entre professores do ensino médio e instituições de ensino, sendo ideal para constituir um laboratório de ciências.
\end{abstract}

Palavras-chave: fotogate, experimento de baixo custo, momento de inércia, intervalo de tempo.

This paper presents the development and validation of a low cost instrument that can be used for time interval measurements, and in other physics experiments. It is proposed to build a photogate combining the simplicity and economy, making it simpler and cheaper than those on the market. The construction of this instrument is based on the use of a light-dependent resistor (LDR) and a LASER pointer, with simple and elementary connections, which characterizes it as a low-cost measuring instrument. Its validation was carried out by comparing the results of the moment of inertia of a homogeneous and thin bar and the experiments carried out in a laboratory, proving its effectiveness as a time variation collection instrument. It is also proposed an experimental activity, whose objective is to determine the landing point of a sphere thrown horizontally from the top of a table at any speed. Its use tends to facilitate the diffusion and access to this type of instrument of measures between high school teachers and educational institutions, being ideal to constitute a science laboratory.

Keywords: photogate, low cost experiment, moment of inertia, time interval.

\section{Introdução}

Em várias atividades experimentais existe a necessidade de se aferir intervalos de tempo, pois se trata de uma grandeza básica, fundamental e importante na caracterização de vários fenômenos físicos e nas medidas de grandezas derivadas tais como velocidade e aceleração. Esta proposta difere de outros trabalhos que propõem soluções alternativas para medidas de intervalo de tempo, como as propostas apresentadas nas Refs. [1-5], pois alia a simplicidade e o baixo custo, sendo de fácil construção e manuseio.

O aparato experimental proposto nesse trabalho tratase de um Fotogate de baixo custo (FBC), caracterizado por ser um instrumento utilizado para indicar a variação de tempo entre um determinado evento. Ele consiste basicamente de um resistor dependente de luz (LDR) e

*Endereço de correspondência: josueama@gmail.com um apontador LASER (light amplification by stimulated emission of radiation, ou seja, amplificação da luz por emissão estimulada de radiação). Utiliza-se a entrada de áudio do computador, combinado com o software livre Audacity [6], diferenciando-se, portanto do dispositivo sugerido na Ref. [7], que utiliza a porta de jogos do PC para fazer aquisição de dados.

Alguns trabalhos, como os desenvolvidos pelas Refs. [8, 9], sugerem a utilização do software Tracker para análise e medidas de intervalos de tempo, com o inconveniente de necessitar de equipamentos de filmagens e análise de imagens. Já o aparato experimental apresentado na Ref. [10] consiste em um sistema de aquisição de dados automático formado por uma chave óptica ativada por luz infravermelha, ligada a um cronômetro digital.

O Fotogate é adequado para ser usado nas instituições de ensino, pois permite variadas adaptações em aulas de física, atendendo assim a todos os níveis de ensino, desde 
a educação básica, à graduação, e também em pesquisas relacionadas com a iniciação científica. Nesse sentido, o FBC sugerido neste trabalho, diferencia-se dos aparatos experimentais desenvolvidos nas Refs. [11, 12], pois é de fácil construção e manuseio, tornando-se prático a coleta dos dados de intervalo de tempo.

O Fotogate sugerido nesse trabalho é inovador, pois sua simplicidade de construção e baixo custo, possibilita ao professor da educação básica construí-lo, dispensando a compra de kits didáticos dispendiosos. Nesse sentido, a concepção do sistema de aquisição de dados passa a ser acessível ao professor da educação básica, que poderá elaborar aulas mais motivadoras para seus alunos utilizando experimentos por meio de aquisição automática de dados com o FBC, bem como as atividades experimentais sugeridas nesse trabalho.

\section{O momento de inércia}

O momento de inércia (I) de um corpo em relação a um eixo, nos remete à necessidade de, nos estudos sobre rotação, recordar o princípio da inércia estudado nos movimentos de translação durante as aplicações das Leis de Newton. Na translação, observa-se que a massa de um objeto se apresenta como o principal agente de inércia. Já na rotação, é preciso verificar como a massa desse objeto se comporta dependendo de sua distribuição em relação ao eixo sobre o qual esse objeto encontra-se em repouso ou sofre rotação.

$\mathrm{Na}$ translação, o princípio da inércia certifica a tendência de um corpo em permanecer no seu estado de repouso ou de movimento. Então, observa-se que quanto maior a massa desse corpo maior a dificuldade em provocar-lhe uma aceleração ou uma desaceleração. De forma correlata, observa-se o efeito do momento de inércia na rotação, como uma medida de resistência que um objeto oferece a alterações no seu estado de movimento de rotação, sendo que essa medida depende da posição em que o eixo se situa em relação ao centro de massa do objeto usado como pêndulo [13].

Quando um corpo rígido não pode ser representado por massas puntiformes, pode-se determinar o momento de inércia considerando-se pequenos elementos de massa $d m$ de modo que todos os pontos encontram-se a uma distância $r$, perpendicular ao eixo de rotação [14]. Então o momento de inércia pode ser expresso pela integral

$$
I=\int r^{2} d m
$$

Escolhendo como elemento de massa $d m$, uma secção reta de comprimento $d x$ situada a uma distância $x$ do ponto $O$, como pode se ver na Fig. 1 . A razão entre o elemento de massa $d m$ e o total de massa $\mathrm{M}$ deve ser igual à razão entre o elemento de comprimento $d x$ e o comprimento total $L$ :

$$
\frac{d m}{M}=\frac{d x}{L} \quad \therefore \quad d m=\frac{M}{L} d x
$$

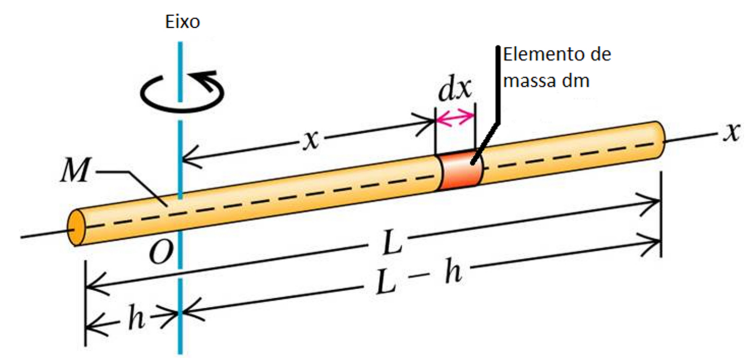

Figura 1: Determinação do momento de inércia de uma barra delgada. Fonte: extraído de [14].

Substituindo $d m$ na equação (1), sendo a distância $x$ e integrando, considerando-se os limites de integração apropriados no eixo ox, obtém-se:

$$
\begin{gathered}
I=\int x^{2} d m=\frac{M}{L} \int_{-h}^{L-h} x^{2} d x=\left[\frac{M}{L}\left(\frac{x^{3}}{3}\right)\right]_{-h}^{L-h} \\
I=\frac{1}{3} M\left[L^{2}-3 L h+3 h^{2}\right]
\end{gathered}
$$

Que é o momento de inércia de uma barra delgada uniforme de massa $M$ e comprimento $L$, com eixo de rotação não coincidente com seu centro de massa. Em que $h$ é a distância entre o eixo de rotação e uma das extremidades.

\section{O Resistor LDR}

O resistor dependente de luz (LDR) é um componente eletrônico com dois terminais que altera a sua resistência de acordo com a variação da intensidade de luz. Também chamados de fotoresistores, apresentam um valor de resistência elevada em um ambiente escuro, e quando expostos à luz, têm a condutividade aumentada. Ou seja, oferecem baixa resistência elétrica quando iluminados.

Optou-se neste trabalho por construir um Fotogate de baixo custo, tendo por base um LDR. O LDR não tem polaridade, ou seja, pode-se ligar seus terminais de qualquer forma. Na Fig. 2 têm-se os símbolos mais usuais para este componente, sendo a Fig. 2a, o mais usual.

\subsection{Funcionamento de um LDR}

O LDR é fabricado com material de alta resistência, como por exemplo o Sulfeto de Cádmio (CdS) ou o Sulfeto de

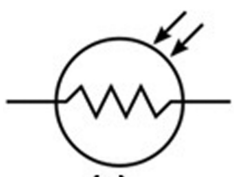

(a)

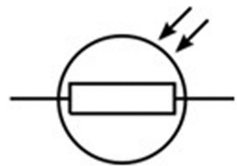

(b)
Figura 2: Esquema de um LDR. Fonte: acervo dos autores. 
Chumbo ( $\mathrm{PbS}$ ), que são materiais semicondutores, dispostos num traçado sinuoso na superfície do componente, possuindo propriedade de diminuir sua resistência à passagem da corrente elétrica quando a luminosidade sobre ele aumenta. Esses materiais possuem poucos elétrons livres quando colocados em ambiente escuro, e liberam elétrons quando há incidência de luz sobre eles, aumentando assim, a condutividade. Este efeito é conhecido como fotocondutividade [15].

Quando cessa a incidência de luz sobre o componente, os elétrons retornam à camada de valência e a resistência do material volta a aumentar. No geral, os LDRs possuem resistências entre $1 \mathrm{M} \Omega$ e $10 \mathrm{M} \Omega$, quando em ambiente escuro, e resistências que podem chegar a menos de 100 $\Omega$ quando iluminados [16].

Alguns LDRs são projetados para responder em luz infravermelha (como os de Sulfito de Chumbo - PbS) ou ainda em luz ultravioleta. A Fig. 3 mostra a estrutura física de um LDR.

\subsection{Curva característica de resistência de um LDR}

O gráfico da Fig. 4 indica a curva característica de resistência de um LDR em relação à quantidade de luz incidente sobre ele, medida em lux. Um lux corresponde a um watt por metro quadrado $\left(1 \mathrm{lux}=1 \mathrm{~W} / \mathrm{m}^{2}\right)$.

Percebe-se, na Fig. 4 que a resistência muito alta deste componente em ambiente com baixa luminosidade, da ordem de $10 \mathrm{M} \Omega$, se reduz a algumas dezenas ou centenas de Ohms quando ele é iluminado diretamente, em um ambiente com intensidade luminosa alta.

\subsection{Espectro de luz}

A variação da resistência do LDR também depende do comprimento de onda da radiação que atinge o componente. Na Fig. 5, pode-se ver um gráfico de resposta espectral de um LDR típico.

Nota-se no gráfico da Fig. 5 que a resposta relativa do LDR considerado é muito maior entre cerca de $530 \mathrm{~nm}$ e $580 \mathrm{~nm}$, ou seja, ele responde muito melhor à variação de luminosidade nessa faixa de comprimento de onda.

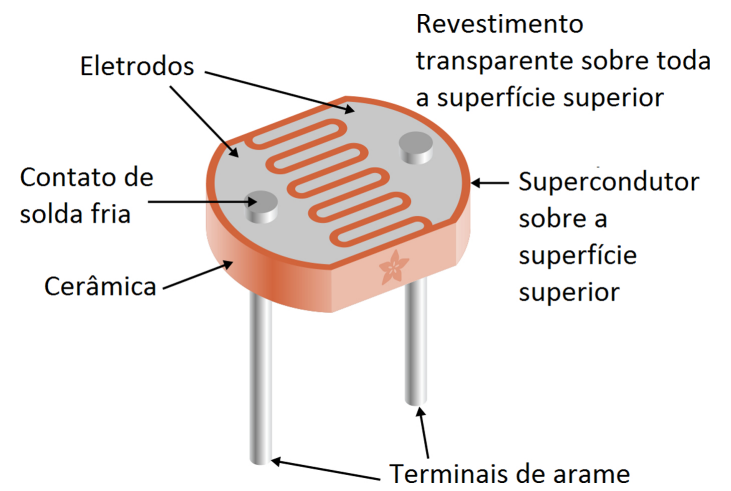

Figura 3: Estrutura física de um LDR. Fonte: acervo dos autores.

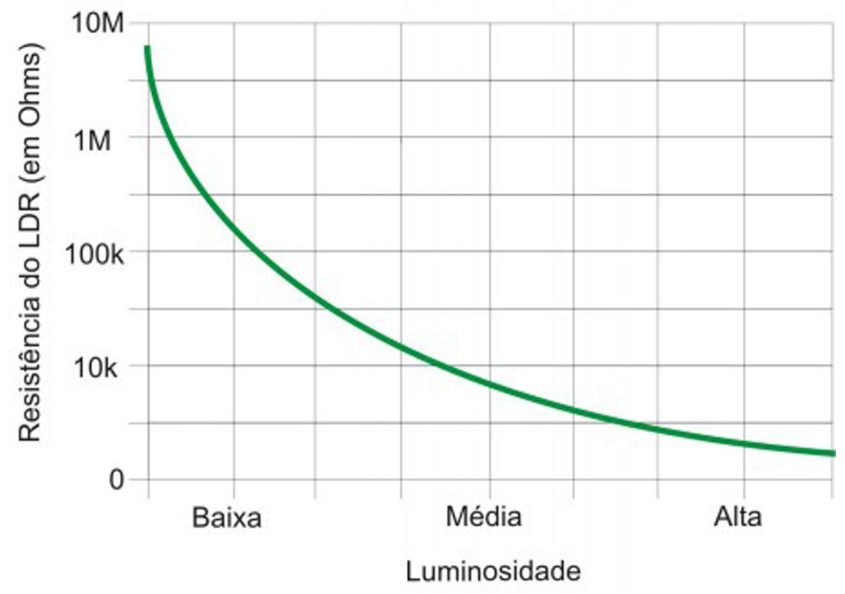

Figura 4: Curva característica do LDR. Fonte: acervo dos autores.

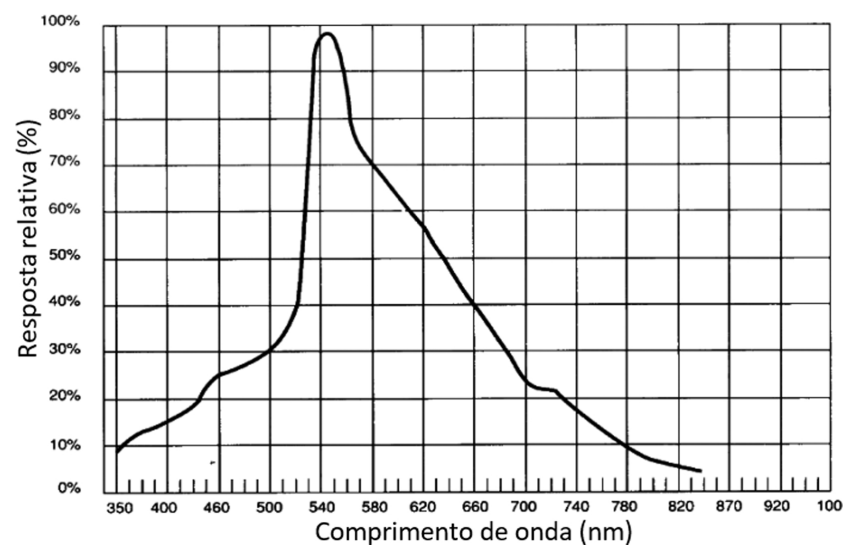

Figura 5: Resposta espectral de um LDR típico. Fonte: acervo dos autores.

\subsection{Tempo de resposta}

O LDR possui um tempo de resposta ou tempo de latência para que a resistência deste componente se estabilize. Esse tempo de recuperação é dado em $\mathrm{K} \Omega / \mathrm{s}$, sendo seu valor típico da ordem de $200 \mathrm{~K} \Omega / \mathrm{s}$, quando se passa de um ambiente iluminado para um ambiente escuro. Quando se passa de um ambiente escuro para iluminado, o tempo de recuperação é da ordem de $10 \mathrm{~ms}$ para que o LDR atinja uma baixa resistência. Esse fator deve ser levado em consideração ao projetar dispositivos que utilizam LDR para acionar equipamentos utilizando a luz ambiente, por exemplo.

A preferência por utilizar-se um diodo LASER na faixa do vermelho, cujo comprimento de onda encontra-se entre $600 \mathrm{~nm}$ e $750 \mathrm{~nm}$ está relacionado, de acordo com o gráfico da Fig. 5, à grande variação da intensidade luminosa, quando este é bloqueado por um objeto opaco. A resposta relativa, que varia em relação ao comprimento de onda, não foi levada em consideração, pois o que se pretende aferir nos experimentos propostos com a utilização do 
Fotogate é o intervalo de tempo entre dois eventos, o que não é afetado pela latência.

\section{A construção do Fotogate de Baixo Custo (FBC)}

A construção do Fotogate de baixo custo (FBC) sugerido neste trabalho é bastante simples, sendo necessários, um LDR de $3 \mathrm{~mm}$, que será usado como oscilador de frequência, sendo responsável por caracterizar a interação com a luz emitida pelo diodo LASER e sua interrupção ocasionada por um objeto opaco, um metro de fios paralelos (cabo AWG40 preto/vermelho) para se efetuar as conexões, um apontador LASER vermelho e um pino P2 mono. Além de um prendedor de roupas, um parafuso, um cotovelo de PVC, um tê de PVC, três pedaços de tubo de PVC de $8 \mathrm{~cm}$ e um pedaço de tubo de PVC de $10 \mathrm{~cm}$. Todos os materiais de PVC devem ter diâmetro de meia polegada ou $20 \mathrm{~mm}$.

A Fig. 6 mostra o esquema de ligação do apontador LASER (6a) e do LDR ligado no pino P2 mono (6b).

Para facilitar o trabalho do professor, foi disponibilizado no canal do You Tube https://www.youtube. com/channel/UC9cQmNxVMELg2A0_p97reMA um vídeo tutorial explicativo de como conceber, montar e utilizar o FBC, bem como sugestões de experimentos baseado na sua aplicação.

Inicialmente deve-se cortar os tubos de PVC nas medidas indicadas anteriormente, sendo que dois deles devem ser furados como demonstrado na Fig. 7b e Fig. 7c, tornando assim possível afixar o parafuso e o LDR.

Após passar o cabo pelos tubos, deve-se soldar o fio no LDR, e depois isolá-los. Deve-se também soldar o

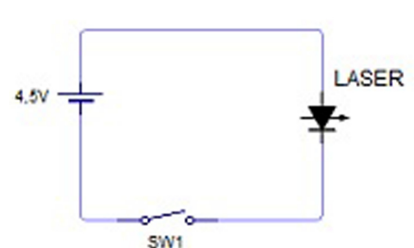

(a) (b)

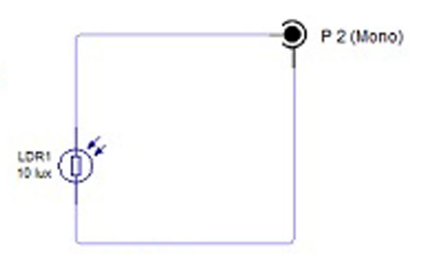

Figura 6: Esquema de ligação do LASER (a) e do LDR (b). Fonte: acervo dos autores.
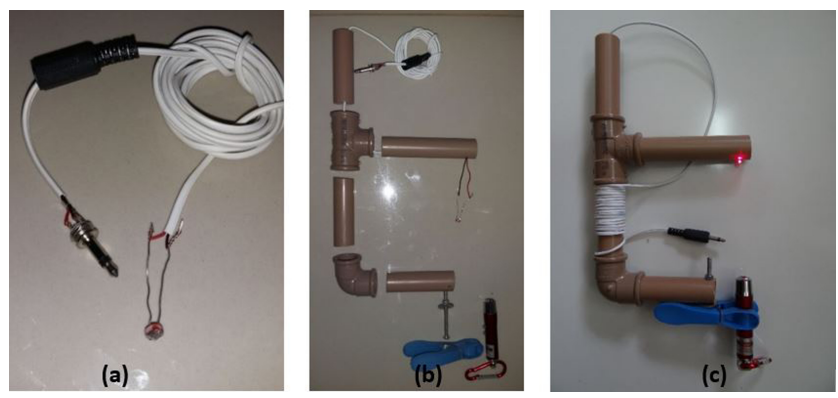

Figura 7: Montagem do FBC. Fonte: acervo dos autores. fio no Pino P2 mono, como pode-se ver na Fig 7a e 7b. Finaliza-se a montagem do FBC encaixando os tubos e fixando o prendedor de roupas por meio do parafuso, como pode-se ver na Fig. 7c.

Para utilizar o FBC, basta conectar o Pino P2 mono na entrada de áudio do PC, com o software Audacity previamente instalado [6]. Na Fig. 8, pode-se ver a interface do FBC, com um notebook, que pode ser substituído por um computador de bancada, e o software Audacity em uso.

\section{Medidas do período de um pêndulo}

Para validar o FBC, utilizou-se uma barra homogênea e delgada como pêndulo físico. O aparato experimental construído para a validação do Fotogate passou pela montagem de um pêndulo físico e a determinação de seu momento de inércia, utilizando-se para isso a equação do período de oscilação desse tipo de pêndulo.

$$
T=2 \pi \sqrt{\frac{I_{o}}{m g d}}
$$

Na equação (3), T é o período de oscilação do pêndulo físico medido com o auxílio do Fotogate, $I_{0}$ é o momento de inércia da barra homogênea, $m$ representa a massa total da barra, $g$ a aceleração da gravidade local e $d$, indica a distância entre o eixo de rotação e o centro de massa da barra.

Manipulando a equação (3), isolando o momento de inércia $I_{0}$, obtém-se a equação (4):

$$
I_{o}=\frac{T^{2} \cdot m \cdot g \cdot d}{4 \pi^{2}}
$$

Com o auxílio do Fotogate e com a interface de coleta de tempo do software livre Audacity (Fig. 9), encontrouse o período de oscilação da barra para cinco medidas realizadas com amplitudes pequenas (Tabela 1), cerca de $10^{\circ}$ em relação a vertical.

De posse dos dados, obtém-se o momento de inércia da barra delgada (Fig. 10):

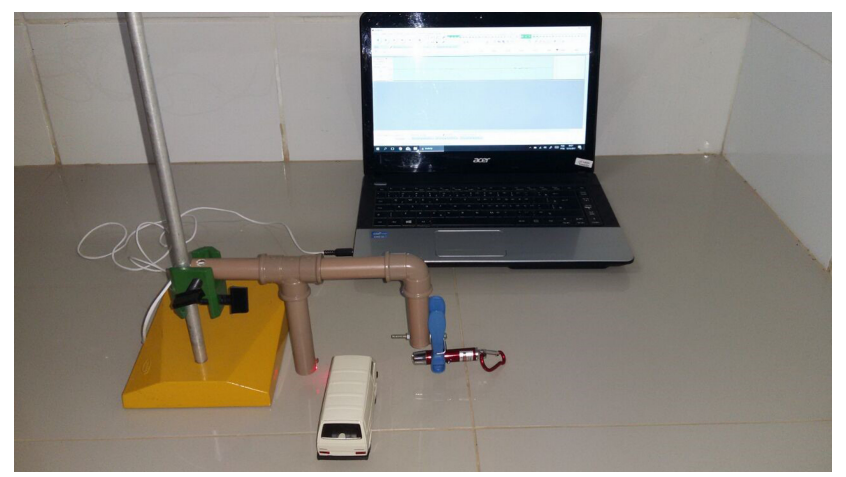

Figura 8: Interface do $\mathrm{FBC}$ conectado à entrada de áudio do PC e o software Audacity. Fonte: acervo dos autores. 


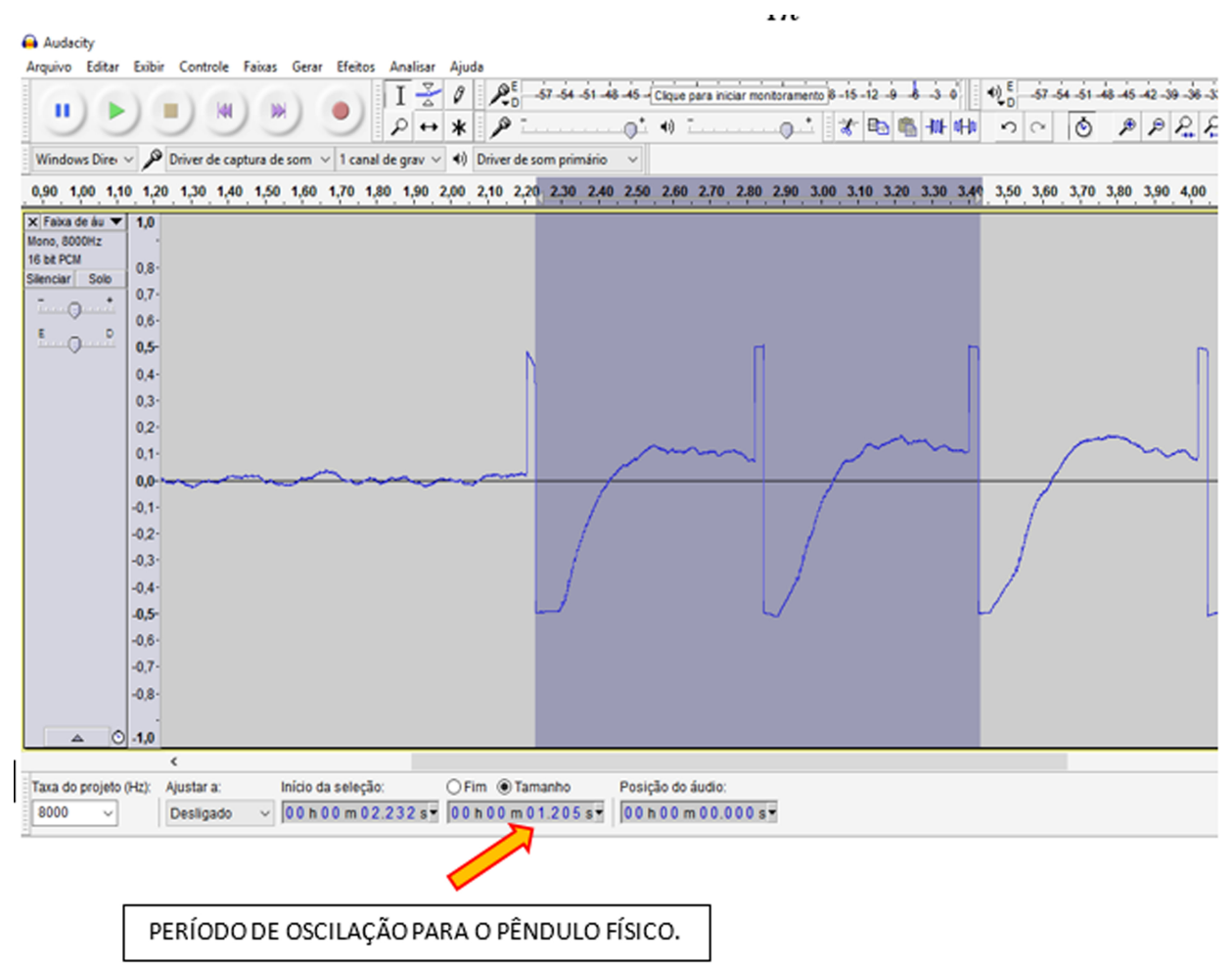

Figura 9: Sinal no detector gerado pela oscilação do pêndulo entre o LDR e a ponteira LASER, indicando os bloqueios e desbloqueios sucessivos produzidos, obtidos no software livre Audacity. Fonte: acervo dos autores.

$$
\begin{aligned}
& T=1,209 \mathrm{~s} \\
& m=62,0 \mathrm{~g}=0,062 \mathrm{~kg} \\
& g=9,820 \mathrm{~m} / \mathrm{s}^{2} \\
& d=20,0 \mathrm{~cm}=0,200 \mathrm{~m}
\end{aligned}
$$

Substituindo os valores em (4) obtém-se:

$$
\begin{gathered}
I_{o}=\frac{T^{2} \cdot m \cdot g \cdot d}{4 \pi^{2}}=\frac{1,209^{2} \times 0,062 \times 9,820 \times 0,200}{4 \pi^{2}} \\
I_{0}=4,51 \times 10^{-3} \mathrm{kgm}^{2}
\end{gathered}
$$

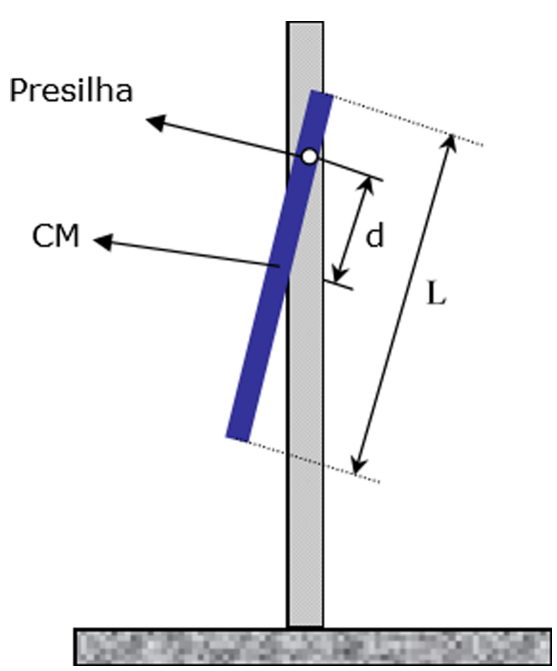

Figura 10: Esquema do arranjo experimental. Fonte: acero dos autores.
Na Fig. 11 pode se ver o arranjo experimental montado para validação do FBC.

Com os dados coletados junto a barra delgada, tem-se: $h=10,0 \mathrm{~cm}=0,100 \mathrm{~m}$

$M=62,0 \mathrm{~g}=0,062 \mathrm{~kg}$

$L=60,0 \mathrm{~cm}=0,600 \mathrm{~m}$

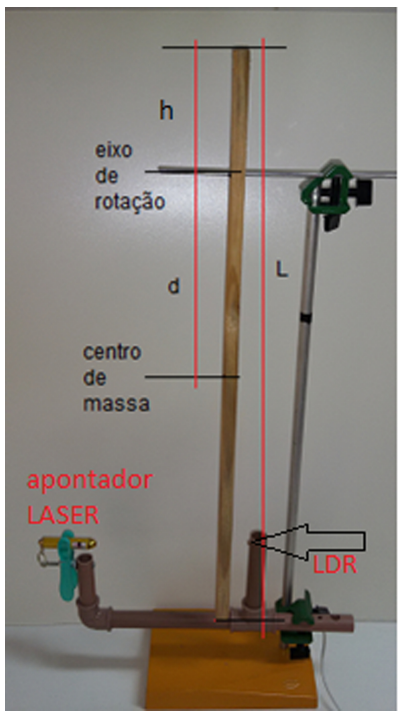

Figura 11: Arranjo experimental. Fonte: acervo dos autores.

Revista Brasileira de Ensino de Física, vol. 40, nº 4, e5403, 2018 
Tabela 1: Medidas realizadas com o FBC. Fonte: resultados obtidos pelos autores

\begin{tabular}{c}
\hline Período (T), em segundos \\
\hline 1,203 \\
1,211 \\
1,213 \\
1,205 \\
1,212 \\
Média $=\mathbf{1 , 2 0 9} \mathbf{~ s}$ \\
\hline
\end{tabular}

Substituindo os valores em 2 obtém-se:

$$
I=\frac{1}{3} M\left[L^{2}-3 L h+3 h^{2}\right]
$$

$$
\begin{gathered}
I=\frac{1}{3} \times 0,062\left[0,600^{2}-3 \times 0,600 \times 0,100+3 \times 0100^{2}\right] \\
I=4,34 \times 10^{-3} \mathrm{kgm}^{2}
\end{gathered}
$$

Os resultados obtidos através da aferição do período do pêndulo físico utilizando-se o FBC e posteriormente do cálculo do momento de inércia da barra utilizando-se das equações (4) e (2), comprovam a robustez do FBC na aferição do intervalo de tempo em experimentos, uma vez que o erro apresentado em valor absoluto foi de apenas $1,7 \times 10^{-4} \mathrm{kgm}^{2}$.

\section{Aplicação: previsão da trajetória de uma esfera}

O FBC proposto neste trabalho, pode ser utilizado em várias atividades experimentais, tais como a situação em que se deseja prever o ponto de aterrissagem de uma esfera lançada horizontalmente do tampo de uma mesa, a uma velocidade qualquer. Para tanto, procede-se à montagem experimental, baseado na Ref. [17], como pode se ver na Fig. 12.

Conhecendo-se a velocidade $v$ da esfera ao deixar a mesa, a altura $(h)$ do tampo da mesa até o chão e a aceleração de queda livre $(g)$, pode-se usar a equação dos movimentos dos projéteis para prever o ponto do chão em que a esfera se aterrissará.

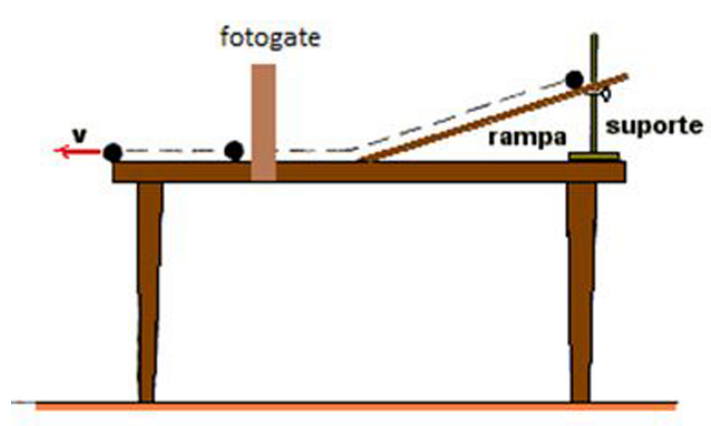

Do movimento dos projéteis, conhece-se sua equação segundo a horizontal,

$$
\Delta x=v \cdot \Delta t
$$

e a equação de queda livre segundo a vertical, a partir do repouso,

$$
h=\frac{1}{2} g \Delta t^{2}
$$

A dificuldade do processo está na medição do intervalo de tempo, que pode ser facilitada com o auxílio do FBC e do software Audacity. A esfera bloqueia a passagem da luz proveniente do apontador LASER, frente ao LDR. Na Fig. 13, têm-se o recorte da tela obtida no software, em que se pode ver a medida do tempo.

Cálculo da velocidade $(v)$ :

$$
v=\frac{d}{\Delta t}
$$

Em que $d=$ diâmetro da esfera $=0,021 \mathrm{me}$

$\Delta t=$ intervalo de tempo da passagem da esfera frente ao laser $=0,029 \mathrm{~s}$

$$
v=\frac{0,021}{0,029}=0,724 \mathrm{~m} / \mathrm{s}
$$

Isolando-se o parâmetro $\Delta t$ em (5) e substituindo em (6), obtêm-se

$$
h=\frac{1}{2} g\left(\frac{\Delta x}{v}\right)^{2}
$$

isolando $\Delta x$ em (8), têm-se:

$$
\Delta x=\sqrt{\frac{2 h}{g}} v
$$

Em que: $\Delta x=$ distância de aterrissagem da esfera em relação à borda da mesa;

$$
\begin{aligned}
& h=\text { altura da mesa ao solo }=0,881 \mathrm{~m} ; \\
& v=0,724 \mathrm{~m} / \mathrm{s}
\end{aligned}
$$

Substituindo esses valores em $\sqrt{9}$, obtêm-se:

$$
\Delta x=0,307 m=30,7 \mathrm{~cm}
$$

O valor encontrado coincide com a medida realizada na atividade experimental, como pode se ver na Fig. 14.

Figura 12: Prevendo o ponto de queda de uma esfera. Fonte: extraído de [17] adaptado. 


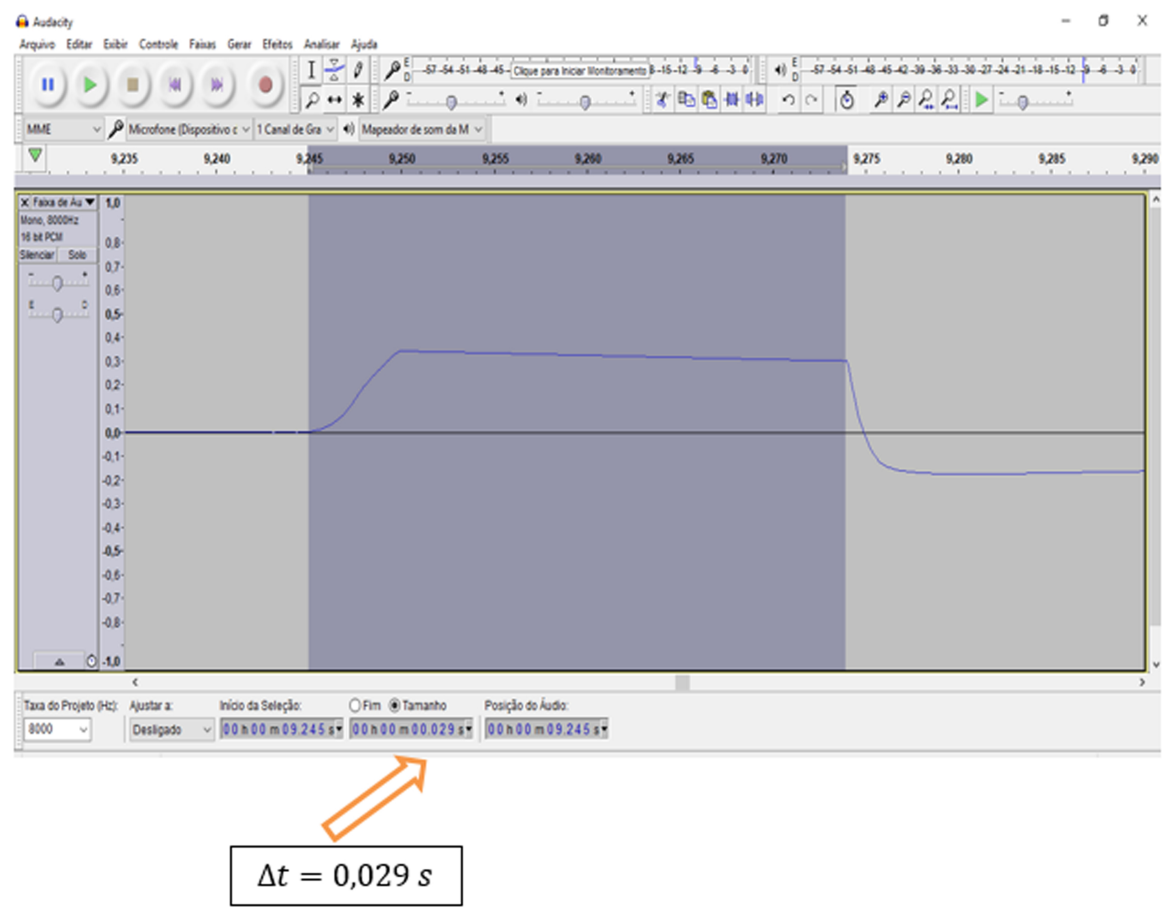

Figura 13: Intervalo de tempo de passagem da esfera em frente ao LASER, detectado com a ajuda do software Audacity. Fonte: acervo dos autores.

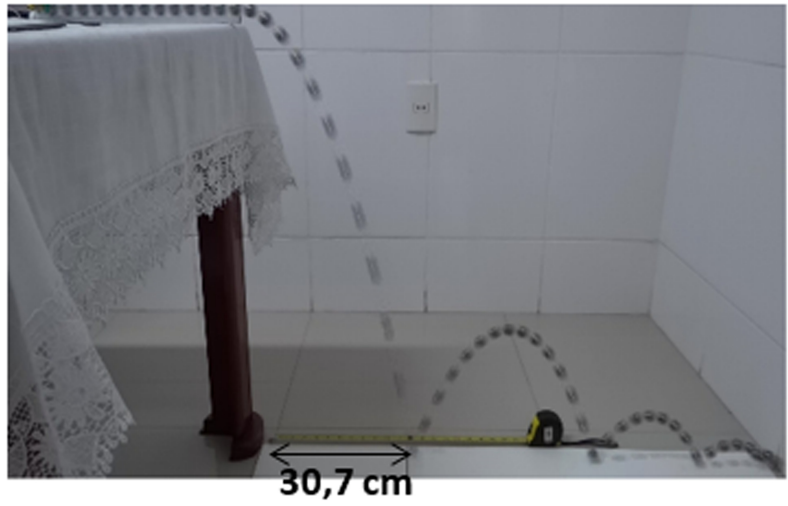

Figura 14: Imagem estroboscópica da esfera em queda. Fonte: acervo dos autores.

\section{Considerações finais}

Proporcionar aos educandos oportunidades de práticas experimentais de física, tornará o ensino mais agradável e significativo. Nesse sentido, a utilização do computador na aquisição de dados é recomendável, principalmente quando se necessita de uma maior precisão nas medidas, ou para diminuir o trabalho mecânico dos educandos.

Nesse sentido o FBC apresentado neste trabalho contribui nos experimentos de física, pois torna-se possível sua utilização numa enorme gama de experimentos, tais como o estudo da queda livre, colisões, períodos do pêndulo simples, a velocidade de um objeto em rolamento e deslizamento, uma vez que permite a coleta de intervalos de tempo, com bastante precisão, facilitando assim a realização de experimentos didáticos em laboratórios de ensino de física, principalmente nas instituições de ensino que não possuem recursos e/ou equipamentos comerciais de alto custo.

\section{Referências}

[1] M. A. Cavalcante e C. R. C. Tavolaro. Revista Brasileira de Ensino de Física 22, 421 (2000).

[2] C. E. Aguiar e F. Laudares. Revista Brasileira de Ensino de Física 23, 371 (2001).

[3] R. Haag. Revista Brasileira de Ensino de Física 23, 176 (2001).

[4] J. S. Figueira e. A. Veit. Revista Brasileira de Ensino de Física 26, 203 (2004)

[5] L. Reyes, B. Buitrago, A. Velasquez, J. Tobón, J. Lopez e J. Otálora. Revista Colombiana de Física 38, 850 (2006).

[6] Audacity, software livre de edição digital de áudio. Disponível para download em: <http://www.audacityteam.org/download/>. Acesso em 13/03/2017.

[7] G. Dionisio e W. C. Magno. Revista Brasileira de Ensino de Física 29 (2007).

[8] J. A. Lenz, N. C. Saavedra Filho e A. G. Bezerra Jr. Abakós 2, 24 (2014).

[9] W. Bonventi Jr. e N. Aranha. Revista Brasileira de Ensino de Física 37, 2504 (2015).

[10] F. F. Luiz, L. E. S. Souza e P. H. Domingues. Revista Brasileira de Ensino de Física 38, 2504 (2016).

[11] H. G. Gutierre, M. S. Ribeiro, L. A. A. Pereira, G. K. Cruz, R. F. Turchiello e S. L. Gómez. Revista Brasileira de Ensino de Física 39, e3501 (2017). 
[12] J. T. Carvalho Neto, F. R. Apolinário e A. A. Soares. Revista Brasileira de Ensino de Física 40, e1504 (2018).

[13] D. Halliday, R. Resnick e J. Walker, Fundamentos de Física: Mecânica (Editora LTC, Rio de Janeiro, 2016), v. $1.10^{\mathrm{a}}$ ed., $372 \mathrm{p}$.

[14] H. D. Young e R. A. Freedman, Sears e Zemansky Física: Mecânica (Pearson, São Paulo, 2016), v. 1. 14 ${ }^{\mathrm{a}} \mathrm{ed}, 448 \mathrm{p}$.

[15] D. Halliday, R. Resnick e J. Walker, Fundamentos de Física: Eletromagnetismo (Editora LTC, Rio de Janeiro, 2016), v. 3. $10^{\mathrm{a}}$ ed., 408 p.

[16] N. C. Braga, Eletrônica Básica para Mecatrônica (Saber, São Paulo, 2005), $1^{\mathrm{a}}$ ed., 160 p.

[17] Feira de Ciências: Previsão das trajetórias. Página pessoal do Prof. Luiz Ferraz Netto. Disponível em: http://www.feiradeciencias.com.br/sala04/ 04_16.asp. Acesso em: 13/03/2018. 